\title{
Bipartite magnetic parent phases in the iron oxypnictide superconductor
}

\author{
M. Hiraishi ${ }^{1}$, S. limura ${ }^{2}$, K. M. Kojima ${ }^{1,3 \star}{ }^{1}$, J. Yamaura ${ }^{4}$, H. Hiraka ${ }^{1}$, K. Ikeda ${ }^{1}$, P. Miao ${ }^{1,3}$, Y. Ishikawa ${ }^{1}$, \\ S. Torii ${ }^{1}$, M. Miyazaki ${ }^{1}$, I. Yamauchi ${ }^{1}$, A. Koda ${ }^{1,3}{ }^{3}$ K. Ishiii ${ }^{5}$, M. Yoshida ${ }^{5,6}$, J. Mizuki $^{6}$, R. Kadono ${ }^{1,3}$, \\ R. Kumai ${ }^{1,3}$, T. Kamiyama ${ }^{1,3}$, T. Otomo ${ }^{1,3}$, Y. Murakami ${ }^{1,3}$, S. Matsuishi ${ }^{4}$ and H. Hosono ${ }^{2,4}$
}

\begin{abstract}
High-temperature superconductivity appears as a consequence of doping charge carriers into an undoped parent compound exhibiting antiferromagnetic order; therefore, ground-state properties of the parent compound are highly relevant to the superconducting state ${ }^{1,2}$. On the basis of this logic, spin fluctuations have been considered as the origin of pairing of the superconducting electrons in the cuprates ${ }^{1}$. As possible pairing mechanisms, there is growing interest in unconventional spin fluctuations or advanced orbital fluctuations owing to the characteristic multi-orbital states in iron pnictides ${ }^{3-6}$. Here, we report the discovery of an antiferromagnetic phase as well as a unique structural transition in electron-overdoped LaFeAsO ${ }_{1-x} \mathrm{H}_{x}(x \sim 0.5)$, whereby a second parent phase is uncovered, albeit heavily doped. The unprecedented two-dome superconducting phases observed in this material can be interpreted as a consequence of the carrier doping starting from the original $x \sim 0$ and additional $x \sim 0.5$ parent phases towards the intermediate region ${ }^{7}$. The bipartite parent phases with distinct physical properties in the second magnetic phase provide us with an interesting example to illustrate the intimate interplay between the magnetic interaction, structural change and orbital degree of freedom in iron pnictide superconductors.
\end{abstract}

Iron pnictides comprise a new class of high-temperature (high- $T_{\mathrm{c}}$ ) superconductors, which has been extensively studied since the discovery of the iron oxypnictide LaFeAsO (La1111; refs 8-14). The superconductivity appears as a result of carrier doping to the parent compound in place of the magnetic- and structural-ordered state $^{2,9,15}$. A recent study reported an advanced doping method using a hydrogen anion instead of fluorine in La1111 that has surpassed the doping limit of fluorine, and uncovered the concealed second superconducting phase (SC2) with a higher $T_{\mathrm{c}}$ of $36 \mathrm{~K}$ at $x \sim 0.35$, in addition to the first dome (SC1) with the maximum $T_{\mathrm{c}}$ of $26 \mathrm{~K}$ at $x \sim 0.1$ (refs 7,16). To investigate the origin of the two SC domes as well as the origin of the higher $T_{c}$ in SC2, and to determine whether a certain hidden phase exists beyond the SC2 region, we have performed a multi-probe study in the range $0.40 \leq x \leq 0.51$ using neutron, muon and $\mathrm{X}$-ray beams.

Figure 1a shows the neutron powder-diffraction pattern in the non-superconducting specimen with $x=0.51$. Extra peaks were observed with indices $(1 / 2,1 / 2, n)_{\mathrm{T}, \mathrm{M}}(n=0,1,2)$ in the low-temperature region, where the subscripts $\mathrm{T}$ and $\mathrm{M}$ refer to the tetragonal cell and magnetic peak, respectively; these were determined to be unambiguously magnetic in origin because they were unobservable in the X-ray measurement. The $(1 / 2,1 / 2,0)_{T, M}$ reflection gradually gains intensity below the magnetic transition temperature $T_{\mathrm{N}}=89(1) \mathrm{K}$, estimated by the power law in the $x=0.51$ sample, as shown in Fig. $1 \mathrm{~b}$, which indicates a continuous second-order transition. Similar magnetic peaks were also observed below $T_{\mathrm{N}} \sim 76 \mathrm{~K}$ for $x=0.45$.

The in-plane magnetic structure is illustrated in the inset of Fig. $1 b^{17}$. The ordering wavevector $\mathbf{q}=(1 / 2,1 / 2,0)$ was determined to correspond to the magnetic unit cell of $\sqrt{ } 2 \mathbf{a}_{\mathrm{T}} \times \sqrt{ } 2 \mathbf{b}_{\mathrm{T}} \times \mathbf{c}_{\mathrm{T}}$, describing a tetragonal axis, which is consistent with the orthorhombic nuclear unit cell in the low-temperature case. The Fe spins are directed to a diagonal axis of the tetragonal cell, and form an antiferromagnetic collinear structure; this result reveals an exceptional stripe-type arrangement among the iron pnictides ${ }^{18-21}$. The spins couple ferromagnetically along the $c$ axis. The magnetic moment in $x=0.51$ at $10 \mathrm{~K}$ is estimated to be $1.21(1) \mu_{\mathrm{B}}$ per iron atom, which is significantly larger than the value of $0.63 \mu_{\mathrm{B}}$ for $x=0$ (ref. 19). This observation that the long-range magnetic order emerges beyond SC2 is a remarkable finding because the $d$-electrons on the iron atoms should be delocalized by the large amount of carrier doping and, accordingly, the magnetic interaction is usually expected to be weak.

Figure $2 \mathrm{a}$ shows the muon spin relaxation ( $\mu \mathrm{SR})$ time spectra for $x=0.45$. The slow relaxation above $80 \mathrm{~K}$ is attributed to a paramagnetic state, whereas the line shapes below $80 \mathrm{~K}$ evolve into strongly damped oscillations with increasing amplitude on cooling, reflecting the development of the magnetically ordered volume fraction (MVF). The significant damping of the muon spin precession in contrast to a clear precession in $x=0$ (ref. 22) indicates an inhomogeneity in the local magnetic fields, which is presumably due to the heavy doping effect (Supplementary Information).

Figure $2 \mathrm{~b}$ is a diagram of the $\mu$ SR process. The amplitude and frequency of the $\mu \mathrm{SR}$ signal reflect the MVF and the ordered moment of the magnetic region, respectively. Figure $2 \mathrm{c}$ shows the temperature dependence of the resultant MVF. For $x=0.51$, the magnetic ordering appears at $T_{\mathrm{N}}=92(7) \mathrm{K}$ and the MVF becomes entire towards zero temperature. As the hydrogen content is reduced, the value of $T_{\mathrm{N}}$ and the MVF decrease in unison. Figure $2 \mathrm{~d}$ shows the MVF and the superconductivity volume fraction (SVF) estimated from susceptibility measurements ${ }^{7}$, clearly indicating an

\footnotetext{
${ }^{1}$ Institute of Materials Structure Science, High Energy Accelerator Research Organization (KEK), Tsukuba, Ibaraki 305-0801, Japan, ${ }^{2}$ Materials and Structures Laboratory, Tokyo Institute of Technology, Yokohama, Kanagawa 226-8503, Japan, ${ }^{3}$ Department of Materials Structure Science, The Graduate University for Advanced Studies, Tsukuba, Ibaraki 305-0801, Japan, ${ }^{4}$ Materials Research Center for Element Strategy, Tokyo Institute of Technology, Yokohama, Kanagawa 226-8503, Japan, ${ }^{5}$ SPring-8, Japan Atomic Energy Agency, Sayo, Hyogo 679-5148, Japan, ${ }^{6}$ School of Science and Technology, Kwansei Gakuin University, Sanda, Hyogo 669-1337, Japan. *e-mail: kenji.kojima@kek.jp
} 

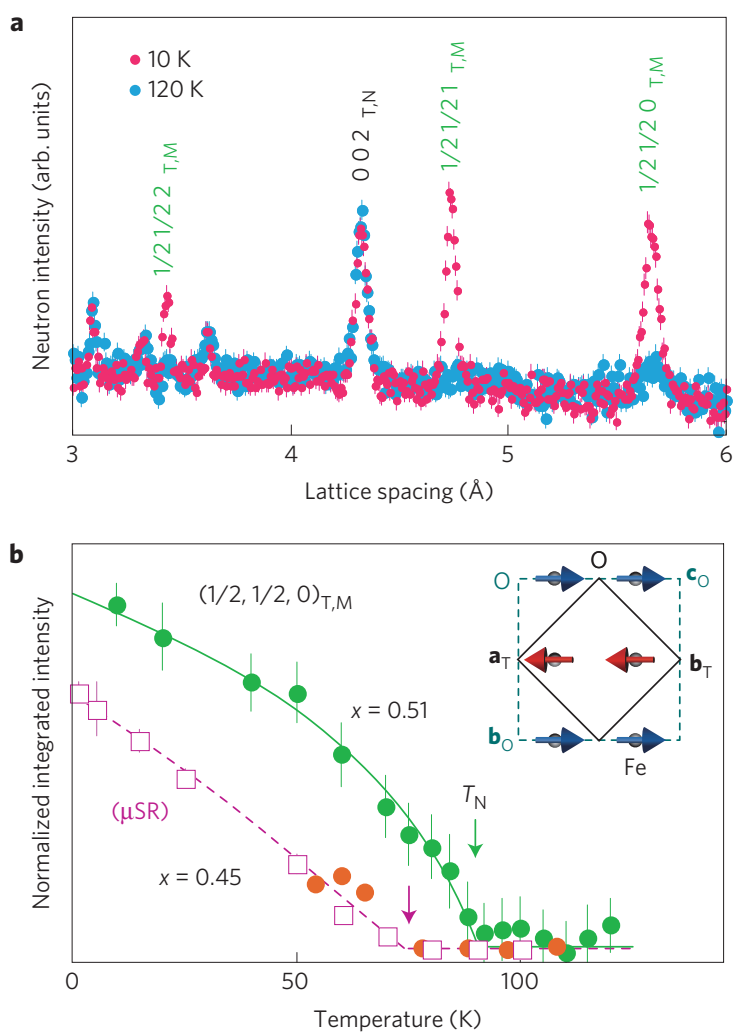

Figure 1 | Pulsed neutron diffraction measurements of $\mathrm{LaFeAsO}_{1-{ }_{-}} \mathrm{H}_{\mathbf{x}}$. We prepared $\sim 1 \mathrm{~g}$ powder samples as described in ref. 7 (Supplementary Information). The powder diffraction measurements were performed on the low-angle detector bank of the Super HRPD at J-PARC for $x=0.51$ and on the NOVA at J-PARC for $x=0.45$. a, Magnetic reflections of $(1 / 2,1 / 2, n)_{T, M}$ $(n=0,1,2)$ that were observed clearly at $10 \mathrm{~K}($ for $x=0.51)$ (labelled in green) disappeared at $120 \mathrm{~K}$. b. Temperature dependence of the integrated intensity of $(1 / 2,1 / 2,0)_{T, M}$ in $x=0.45$ (orange) and 0.51 (green), normalized to the nuclear $(0,0,2)_{T, N}$ reflection (labelled in black in Fig. 1a). The data for $x=0.51$ were fitted to the power law $\left(T_{N}-T\right)^{2 \beta}$, resulting in $T_{N}=89(1) \mathrm{K}$ and $2 \beta=0.58(5)$. The squared order parameter for $x=0.45$ determined by $\mu \mathrm{SR}$ ( $\alpha$ (muon frequency $)^{2} \times$ (magnetic volume fraction)) is plotted (purple) after scaling to neutron data. The in-plane configuration of the Fe spin, determined by the Rietveld method ${ }^{17}$, for $x=0.45$ as well as 0.51 , is illustrated in the inset with the tetragonal unit cell $\mathbf{a}_{\mathrm{T}} \times \mathbf{b}_{\mathrm{T}}$ (solid line) in the high-temperature case and the magnetic unit cell $\sqrt{ } 2 \mathbf{a}_{T} \times \sqrt{ } 2 \mathbf{b}_{T}$ (broken line) in the low-temperature case. The error bars represent the statistical error in a and the uncertainty in the least-squares fitting of the peaks in $\mathbf{b}$

inverse correlation between the MVF and the SVF. The approximate summation to unity suggests that antiferromagnetic static order and superconductivity coexist in the range $0.40 \leq x \leq 0.45$ despite the absence of the coexistence state in the underdoped region $^{15}$. The coexistence state is not attributed to the microscopic scale but to the mesoscopic scale in the spatial inhomogeneity, because if the coexistence state were due to the microscopic scale the MVF obtained by the $\mu S R$ experiment would become entire even in the superconducting state (Supplementary Information). Further experimental work will clarify any intimate connection between superconductivity and magnetism in the heavier doping region ${ }^{23,24}$.

Figure $3 \mathrm{a}$ shows the X-ray profiles of the $(2,2,0)_{\mathrm{T}}$ reflections in the $x=0.45$ and 0.51 samples. On cooling, the peak in the $x=0.51$ sample was broadened, whereas the peak in $x=0.45$ exhibited only slight broadening. No broadening of the $(0,0, l)$ reflections was observed in the $x=0.51$ sample; therefore, the experimental findings imply that the tetragonal to orthorhombic (T-O) structural transition emerges clearly for $x=0.51$.

Figure $3 \mathrm{~b}$ shows the temperature dependence of the lattice constants in samples with $x=0.45$ and 0.51 . In $x=0.51$, the $a_{\mathrm{T}}$ axis length splits in two below the T-O transition of $T_{\mathrm{s}} \sim 95 \mathrm{~K}$, and the $c_{\mathrm{T}}$ axis length shows an upturn at $T_{\mathrm{s}}$. In $x=0.45$, although an apparent split between $a_{\mathrm{T}}$ and $b_{\mathrm{T}}$ does not exist, the $c_{\mathrm{T}}$ axis anomaly appears to be similar to that in $x=0.51$, which may be the result of insufficient coherence of the splitting of $a_{\mathrm{T}}$ and $b_{\mathrm{T}}$ in the low-temperature phase.

The resultant temperature dependence of the structural and magnetic order parameters is shown in Fig. $3 c$ for $x=0.51$. The small gap between the transition temperatures $T_{\mathrm{s}}$ and $T_{\mathrm{N}}$ implies a strong correlation between structure and magnetism in comparison with the large gap of $\sim 20 \mathrm{~K}$ for $x=0$. On the basis of a structural analysis $^{25}$, the compound with $x=0.51$ crystallizes below $T_{\mathrm{s}}$ in an orthorhombic Aem 2 structure without inversion symmetry, in contrast to the universal Cmme structure with inversion symmetry observed in the 1111 materials (Supplementary Information). The $\mathrm{Fe}$ atom exhibits an off-centre deformation in the $\mathrm{FeAs}_{4}$ tetrahedron, as shown in the inset of Fig. 4. The present result provides us with an intriguing deduction that the unusual structural distortion, along with the loss of the inversion symmetry, which has not been found in large ensemble of the iron pnictides ${ }^{18-21}$, may profoundly affect the $d$-orbital levels of the iron atom ${ }^{26,27}$.

Figure 4 shows the phase diagram of $\mathrm{LaFeAsO}_{1-x} \mathrm{H}_{x}$, where the introduction of charge carriers via doping with hydrogen indeed controls the system (Supplementary Information). The features of the physical properties in $x=0.51(x=0)$ can be characterized as follows: the Fe-spin arrangement is a peculiar stripe-type (universal stripe-type $)^{18,19}$; the magnetic moment is large, 1.21(1) $\mu_{\mathrm{B}}$ (small, $\left.0.63 \mu_{\mathrm{B}}\right)$; the gap between $T_{\mathrm{s}}$ and $T_{\mathrm{N}}$ is $\sim 5 \mathrm{~K}(\sim 20 \mathrm{~K})$; the structural symmetry is non-centrosymmetric (centrosymmetric); the magnetic and superconducting states coexist (the magnetic and superconducting states are exclusive); the behaviour of resistivity is as for a non-Fermi liquid (Fermi liquid) ${ }^{7}$. These findings strongly suggest the magnetic and electronic correlations are considerably developed in the right-hand region of the phase diagram.

We have thus far regarded the 'undoped' antiferromagnet with the structural transition adjacent to the superconducting state as the parent compound in the high- $T_{c}$ superconductors. Hence, we now reveal that a newly discovered antiferromagnetic phase with a structural transition adhering to the superconducting phase is considered as the 'doped' parent phase. This behaviour is unexpected, because magnetic and electronic correlations are widely perceived as being weak in the region with heavy carrier doping. However, the magnetic and electronic correlations in the advanced parent phase at $x \sim 0.5$ are indeed rather strong in comparison to the original parent phase at $x \sim 0$. We think that a multi-band feature such as an orbitally selective Hund's coupling or a Mott transition plays a crucial role so as to drive the emergence of the advanced parent phase, which is presumably stimulated by the half-integer number of the $d^{6.5}$ state in $x=0.5$ (refs 28,29). In the iron pnictides, the 'parent compound' not only refers to the undoped material, but also more generally indicates a fingerprint at a certain critical point of the magnetic and electronic correlations. Yamakawa et al. proposed spin and orbital ordered states with the commensurate Fermi surface nesting at $x=0$ and the incommensurate Fermi surface nesting at $x \sim 0.4$ in $\mathrm{LaFeAsO}_{1-x} \mathrm{H}_{x}$ from the theoretical calculation $^{30}$; the latter inconsistency with the present result is presumably due to the sizeable electron correlation with the large magnetic ordered moment. A more sophisticated theory concerning the electron correlation, the multi-band picture, and the heavy doping effect in the crystal is required to specify the origin of the advanced parent phase.

We now discuss the origin of the two-dome SC phases. The electron doping to the $d^{6}$ state of the $x=0$ parent-that is, the 

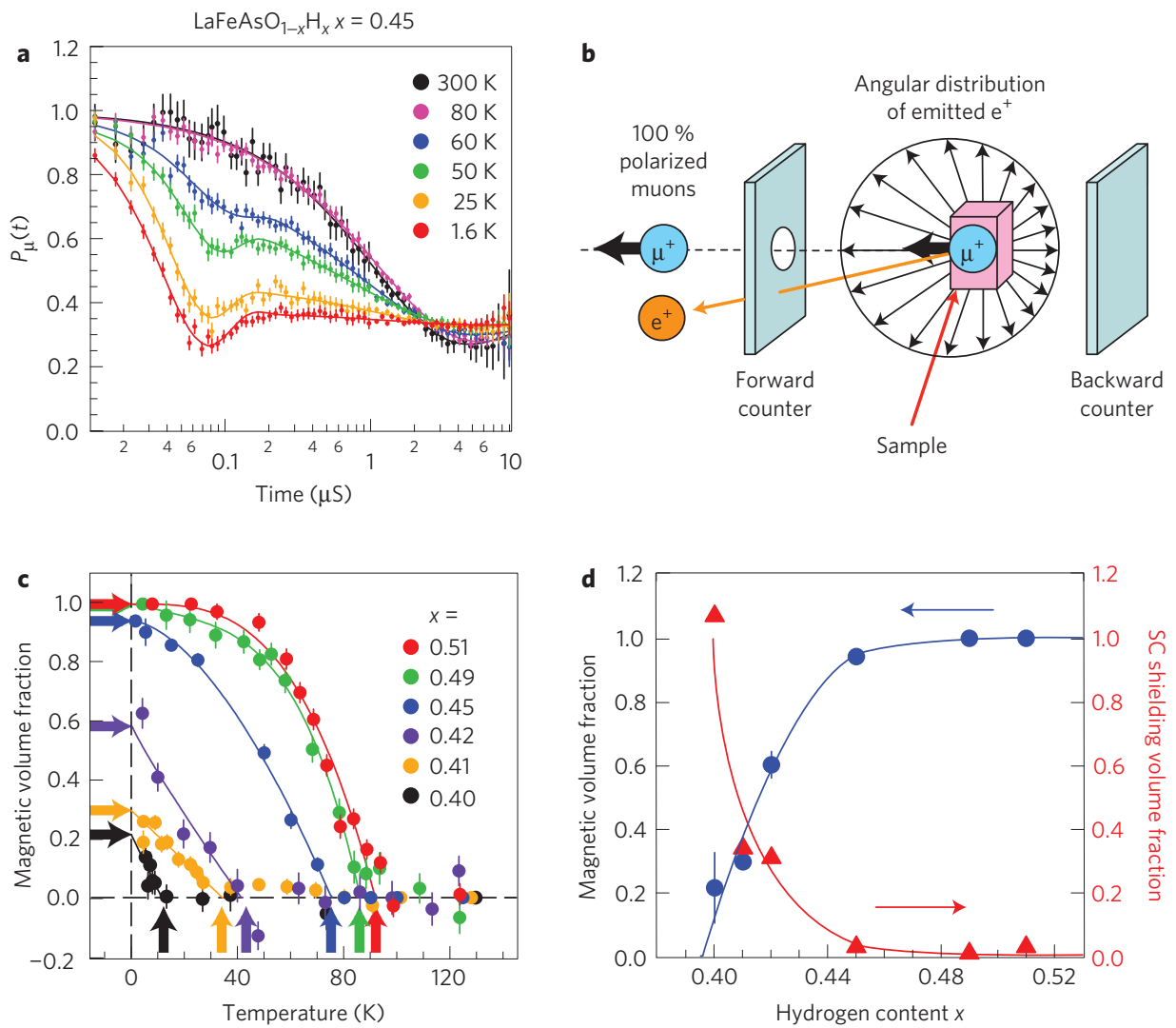

Figure $\mathbf{2}$ | Zero-field muon spin relaxation measurements. a, Time spectra of the muon polarization $P_{\mu}$ for $x=0.45$, taken at PSI. The data exhibit significant damping in contrast to a clear muon spin precession in $x=0$ (ref. 22), indicating an inhomogeneity in the local magnetic fields. $\mathbf{b}$, Diagram of the muon spin relaxation process. The magnetic volume fraction (MVF) is estimated from the amplitude of the time-dependent asymmetry in the positron counts between the forward and backward counters. c, Temperature dependence of the MVF for samples in the range $x=0.40-0.51$, conducted at J-PARC MUSE and PSI. d, Hydrogen concentration dependence of the MVF (blue) and the superconductivity (SC) shielding volume fraction (red). The error bars represent the statistical error in $\mathbf{a}$ and the uncertainty in the least-squares fittings of the spectra in $\mathbf{c}$ and $\mathbf{d}$

a



b

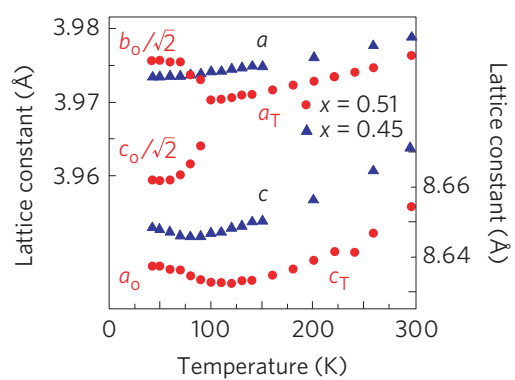

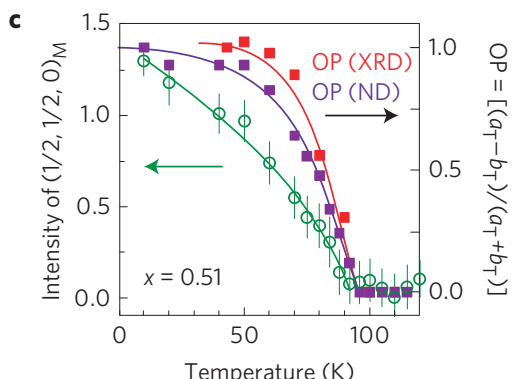

Figure 3 | Synchrotron X-ray diffraction measurements. Powder diffraction measurements for samples with $x=0.42,0.45,0.49$ and 0.51 were performed on the beamlines of $8 \mathrm{~A} / 8 \mathrm{~B}$ at KEK-PF. $\mathbf{a}$, Representative profiles of the $(2,2,0)_{\top}$ reflections for $x=0.45$ and 0.51 at a wavelength of $\lambda=1.0993 \AA$. Peak broadening was observed in $x=0.51$ below $T_{\mathrm{s}} \sim 95 \mathrm{~K}$, whereas there was slight broadening in $x=0.45$. $\mathbf{b}$, The temperature dependence of the lattice constants for $x=0.45$ and 0.51 . $\ln x=0.51$, the $a_{T}$ - and $c_{T}$-axes exhibit a split in length and an upturn with the T-O transition of $T_{\mathrm{s}} \sim 95 \mathrm{~K}$, respectively. In the orthorhombic phase, the values of $b_{O}$ and $c_{O}$ are divided by $\sqrt{ } 2$. The cell setting changes as $\mathbf{a}_{O}=\mathbf{c}_{T}, \mathbf{b}_{\mathrm{O}}=\mathbf{a}_{\mathrm{T}}+\mathbf{b}_{\mathrm{T}}$ and $\mathbf{c}_{\mathrm{O}}=-\mathbf{a}_{\mathrm{T}}+\mathbf{b}_{\mathrm{T}}$ (Fig. $1 \mathrm{~b}$ inset). The orthorhombicity, $\Delta a_{T} / a_{T}=0.41 \%$, is smaller than that of the other iron pnictides $\left(0.49 \%, \mathrm{LaFeAsO} ; 0.73 \%, \mathrm{BaFe}_{2} \mathrm{As}_{2} ;\right.$ refs 18,20$)$. c, Temperature dependence of the structural order parameter (OP) determined by neutron and X-ray diffraction, and the magnetic intensity of the $(1 / 2,1 / 2,0)_{T, M}$ reflection in $x=0.51$. The error bars indicate the uncertainty in the least-squares fitting of the magnetic peaks in $\mathbf{c}$.

chemical substitution of $\mathrm{H}^{-}$to the $\mathrm{O}^{2-}$ site-leads to the SC1 phase. From the viewpoint of the $x \sim 0.5$ parent, the SC2 phase emerges through hole doping to the $d^{6.5}$ state, by $\mathrm{O}^{2-}$ substitution to the $\mathrm{H}^{-}$ site. Consequently, we can definitely state that the two SC domes are generated by carrier doping, starting from the left- and right-hand parent compounds towards the intermediate region of the phase diagram. Moreover, the $T_{\mathrm{c}}$ valley at $x \sim 0.2$ is interpreted as anique crossover region in this phase diagram.
The combination of the distinct feature of the structural instability with the loss of inversion symmetry and the localized nature of the $d$-electrons relative to the original parent compound plausibly result in a new mechanism of superconductivity as the origin of the significant enhancement of $T_{c}$ in SC2 relative to SC1, because the ground-state properties in the parent compounds generally persist unabated, even in the superconducting state. Future band structure calculations taking into account the orthorhombic 


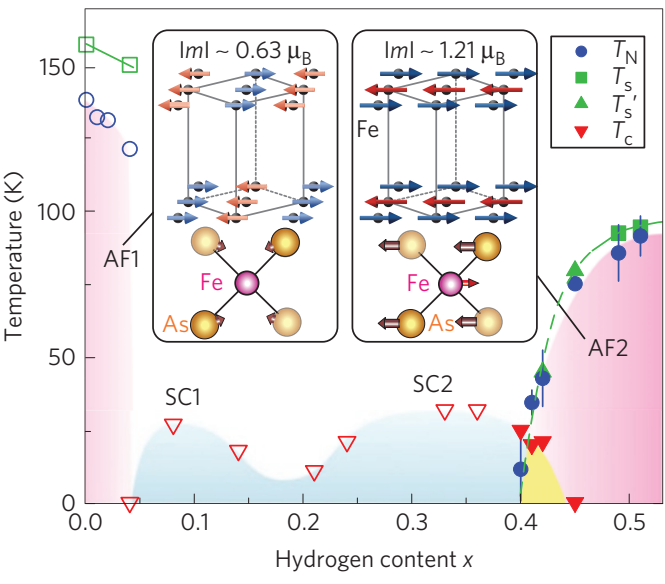

Figure 4 | Magnetic, structural and superconducting phase diagram of LaFeAsO ${ }_{1-x} \mathrm{H}_{x}$. The original parent compound with $x=0$ exhibits a structural transition at $T_{\mathrm{s}}=155 \mathrm{~K}$, followed by an antiferromagnetic state (AF1) at $T_{N}=137 \mathrm{~K}$ (ref. 18). With increasing $x$, two superconductivity domes appear: $0.05 \leq x \leq 0.20(\mathrm{SC} 1)$ with $T_{c, \max }=26 \mathrm{~K}$, and $0.20 \leq x \leq 0.42(\mathrm{SC} 2)$ with $T_{c, \max }=36 \mathrm{~K}$ (ref. 7). Eventually, another antiferromagnetic phase (AF2) appears in the range $0.40 \leq x \leq 0.51$. In the advanced parent compound at $x=0.51$, structural and magnetic transitions occur at $T_{\mathrm{s}} \sim 95 \mathrm{~K}$ and $T_{\mathrm{N}}=89 \mathrm{~K}$, respectively. $T_{\mathrm{s}}{ }^{\prime}$ indicates the $\mathrm{c}$ axis upturn temperature observed in X-ray measurements. The filled and open marks are obtained from the present and previous results, respectively ${ }^{7}$. The magnetic structures of AF1 (left) and AF2 (right) are shown with their magnetic moments $m$ (ref. 19), where the solid lines represent the tetragonal cell. The displacements of the Fe and As atoms across the structural transitions are schematically described by the arrows on the $\mathrm{FeAs}_{4}$ tetrahedra from the view of the orthorhombic long axis, in which the Fe and As atoms move by $0.07 \AA(0 \AA)$ and $0.06 \AA(0.01 \AA)$ in $x=0.51(x=0)$, respectively. The error bars represent the uncertainty in the least-squares fitting routines.

crystal and magnetic structures would confirm and clarify this new ordered state.

Received 21 July 2013; accepted 30 January 2014; published online 16 March 2014

\section{References}

1. Lee, A. P., Nagaosa, N \& Wen, X-G. Doping a Mott insulator: Physics of high-temperature superconductivity. Rev. Mod. Phys. 78, 17-85 (2006).

2. Paglione, J. \& Greene, R. L. High-temperature superconductivity in iron-based materials. Nature Phys. 6, 645-658 (2010).

3. Singh, D. J. \& Du, M-H. Density functional study of $\mathrm{LaFeAsO}_{1-x} \mathrm{~F}_{x}$ : A low carrier density superconductor near itinerant magnetism. Phys. Rev. Lett. 100, 237003 (2008).

4. Kuroki, K. et al. Unconventional pairing originating from disconnected Fermi surfaces of superconducting $\mathrm{LaFeAsO}_{1-x} \mathrm{~F}_{x}$. Phys. Rev. Lett. 101, 087004 (2008).

5. Mazin, I., Singh, D. J., Johannes, M. D. \& Du, M. H. Unconventional superconductivity with a sign reversal in the order parameter of $\mathrm{LaFeAsO}_{1-x} \mathrm{~F}_{x}$. Phys. Rev. Lett. 101, 057003 (2008).

6. Kontani, H. \& Onari, S. Orbital-fluctuation-mediated superconductivity in iron pnictides: Analysis of the five-orbital Hubbard-Holstein model. Phys. Rev. Lett. 104, 157001 (2010).

7. Iimura, S. et al. Two-dome structure in electron-doped iron arsenide superconductors. Nature Commun. 3, 943 (2012).

8. Kamihara, Y. et al. Iron-based layered superconductor: LaOFeP. J. Am. Chem. Soc. 128, 10012-10013 (2006)

9. Kamihara, Y., Watanabe, T., Hirano, M. \& Hosono, H. Iron-based layered superconductor $\mathrm{La}\left[\mathrm{O}_{1-x} \mathrm{~F}_{x}\right] \mathrm{FeAs}(x=0.05-0.12)$ with $T_{\mathrm{c}}=26 \mathrm{~K}$. J. Am. Chem. Soc. 130, 3296-3297 (2008)
10. Chen, X. H. et al. Superconductivity at $43 \mathrm{~K}$ in $\mathrm{SmFeAsO}_{1-x} \mathrm{~F}_{x}$. Nature 453, 761-762 (2008)

11. Takahashi, H. et al. Superconductivity at $43 \mathrm{~K}$ in an iron-based layered compound $\mathrm{LaO}_{1-x} \mathrm{~F}_{x}$ FeAs. Nature 453, 376-378 (2008).

12. Rotter, M., Tegel, M. \& Johrendt, D. Superconductivity at $38 \mathrm{~K}$ in the iron arsenide $\left(\mathrm{Ba}_{1-x} \mathrm{~K}_{x}\right) \mathrm{Fe}_{2} \mathrm{As}_{2}$. Phys. Rev. Lett 101, 107006 (2008).

13. Yildirim, T. Origin of the $150-\mathrm{K}$ anomaly in LaFeAsO: Competing antiferromagnetic interactions, frustration, and a structural phase transition. Phys. Rev. Lett. 101, 057010 (2008).

14. Ma, F. \& Lu, Z-Y. Iron-based layered compound $\mathrm{LaFeAsO}$ is an antiferromagnetic semimetal. Phys. Rev. B 78, 033111 (2008).

15. Luetkens, $\mathrm{H}$. et al. The electronic phase diagram of the $\mathrm{LaO}_{1-x} \mathrm{~F}_{x} \mathrm{FeAs}$ superconductor. Nature Mater. 8, 305-309 (2009).

16. Matsuishi, S. et al. Structural analysis and superconductivity of $\mathrm{CeFeAsO}_{1-x} \mathrm{H}_{x}$. Phys. Rev. B 85, 014514 (2012).

17. Rodriguez-Carvajal, J. Recent advances in magnetic structure determination by neutron powder diffraction. Physica B 192, 55-69 (1993).

18. De la Cruz, C. et al. Magnetic order close to superconductivity in the iron-based layered $\mathrm{LaO}_{1-x} \mathrm{~F}_{x}$ FeAs systems. Nature 453, 899-902 (2008).

19. Qureshi, N. et al. Crystal and magnetic structure of the oxypnictide superconductor $\mathrm{LaFeAsO}_{1-x} \mathrm{~F}_{x}$ : A neutron-diffraction study. Phys. Rev. B 82 184521 (2010).

20. Huang, Q. et al. Neutron-diffraction measurements of magnetic order and a structural transition in the parent $\mathrm{BaFe}_{2} \mathrm{As}_{2}$ compound of FeAs-based high-temperature superconductors. Phys. Rev. Lett. 101, 257003 (2008).

21. Lumsden, M. D. \& Christianson, A. D. Magnetism in Fe-based superconductors. J. Phys. Condens. Matter 22, 203203 (2010).

22. Klauss, H-H. et al. Commensurate spin density wave in LaFeAsO: A local probe study. Phys. Rev. Lett. 101, 077005 (2008).

23. Uemura, Y. J. Superconductivity: Commonalities in phase and mode. Nature Mater. 8, 253-255 (2009).

24. Takeshita, S. et al. Insular superconductivity in a Co-doped iron pnictide $\mathrm{CaFe}_{1-x} \mathrm{Co}_{x}$ AsF. Phys. Rev. Lett. 103, 027002 (2009).

25. Izumi, F. \& Momma, K. Three-dimensional visualization in powder diffraction. Solid State Phenom. 130, 15-20 (2007).

26. Lee, C-C., Yin, W-G. \& Ku, W. Ferro-orbital order and strong magnetic anisotropy in the parent compounds of iron-pnictide superconductors. Phys. Rev. Lett. 103, 267001 (2009).

27. Lv, W., Wu, J. \& Phillips., P. Orbital ordering induces structural phase transition and the resistivity anomaly in iron pnictides. Phys. Rev. B 80, 224506 (2009).

28. de’ Medici, L., Hassan, S. R., Capone, M. \& Dai, X. Orbital-selective Mott transition out of band degeneracy lifting. Phys. Rev. Lett. 102, 126401 (2009).

29. Lanatà, N. et al. Orbital selectivity in Hund's metals: The iron chalcogenides. Phys. Rev. B 87, 045122 (2013).

30. Yamakawa, Y. et al. Phase diagram and superconducting states in $\mathrm{LaFeAsO}_{1-x} \mathrm{H}_{x}$ based on the multiorbital extended Hubbard model. Phys. Rev. $B$ 88, 041106(R) (2013).

\section{Acknowledgements}

We thank K. Yamada for helpful discussions. The neutron, muon and synchrotron radiation experiments were performed at J-PARC (BL08-SuperHRPD, BL21-NOVA, Muon D1), PSI, KEK-PF (BL-8A/8B), and SPring- 8 with the approval of JASRI (BL11XU) (Proposal Nos. 2013S2-002, 2009S05, 2013A3502). This work was supported by MEXT Elements Strategy Initiative to Form Core Research Center.

\section{Author contributions}

Y.M., R. Kadono, S.I., S.M. and H. Hosono conceived the study. M.H., K.K., M.M., I.Y., A.K. and R. Kadono carried out the muon experiment. S.I. synthesized the samples. J.Y. and R. Kumai measured the synchrotron X-ray diffraction. H. Hiraka, J.Y., K. Ikeda, P.M., Y.I., S.T., T.K. and T.O. performed the neutron scattering experiments. J.Y., M.Y. and K. Ishii measured the X-ray absorption. M.H., K.K., J.Y. and H. Hiraka co-wrote the manuscript. All the authors discussed the results and the manuscript.

\section{Additional information}

Supplementary information is available in the online version of the paper. Reprints and permissions information is available online at www.nature.com/reprints. Correspondence and requests for materials should be addressed to K.K.

\section{Competing financial interests}

The authors declare no competing financial interests. 